\title{
GUIDELINES
}

\section{Management of diabetes from preconception to the postnatal period: summary of NICE guidance}

\author{
The Guideline Development Group
}

\section{Moira A Mugglestone (for the} Guideline Development Group), National Collaborating Centre for Women's and Children's Health, London W1T 2QA

mmugglestone@ncc-wch.org.uk

BMJ 2008;336:714-7

doi:10.1136/bmj.39505.641273.AD
This is one of a series of $B M J$ summaries of new guidelines, which are based on the best available evidence; they will highlight important

recommendations for clinical practice, especially where uncertainty or controversy exists. Further information about the guidance, a list of members of the guideline development group, the supporting evidence statements, and additional references are in the longer version of this article on bmj.com.

\section{Why read this summary?}

Diabetes in pregnancy is associated with risks to the woman (for example, higher rates of miscarriage, preeclampsia, and preterm labour) and to the developing fetus and baby (for example, higher rates of congenital malformations, macrosomia, birth injury, and perinatal mortality). ${ }^{12}$ This article summarises the most recent guidance from the National Institute for Health and Clinical Excellence (NICE) on how to manage diabetes and its complications from preconception to the postnatal period. ${ }^{3}$

\section{Recommendations}

NICE recommendations are based on systematic reviews of best available evidence. When minimal evidence is available, recommendations are based on the guideline development group's opinion of what constitutes good practice. Evidence levels for the recommendations are in the longer version of this article on bmj.com.

Preconception information for women with pre-existing diabetes

- From adolescence onwards, advise women about the importance of avoiding unplanned pregnancy.

- Inform women and their families about how diabetes affects pregnancy and how pregnancy affects diabetes (box).

- Offer preconception care and advice to women who are planning to become pregnant before they discontinue contraception; inform them that establishing good glycaemic control before conception and continuing throughout pregnancy reduces-but does not eliminate-the risks of miscarriage, congenital malformation, stillbirth, and neonatal death.

- Offer a structured education programme (such as "dose adjustment for normal eating" (DAFNE) for type 1 diabetes, "diabetes education and self management for ongoing and newly diagnosed" type 2 diabetes (DESMOND), or X-PERT for type 2 diabetes) as soon as possible to women who are planning to become pregnant (unless they have already attended one).

Glycaemic control before and during pregnancy

- Advise women with pre-existing diabetes who are planning to become pregnant to keep their $\mathrm{HbA}_{1 \mathrm{c}}$ concentration below $6.1 \%$ if this is safely achievable; reassure women that any reduction in $\mathrm{HbA}_{1 \mathrm{c}}$ towards the target of $6.1 \%$ is likely to reduce the risk of congenital malformations; women whose $\mathrm{HbA}_{1 \mathrm{c}}$ is above $10 \%$ should be strongly advised to avoid pregnancy.

- Offer monthly measurement of $\mathrm{HbA}_{1 \mathrm{c}}$ to women with pre-existing diabetes who are planning to become pregnant; do not use $\mathrm{HbA}_{1 \mathrm{c}}$ routinely for assessing glycaemic control in the second and third trimesters of pregnancy.

- Agree individualised targets for self monitoring of blood glucose concentration before and during pregnancy, taking into account the risk of hypoglycaemia; advise pregnant women with diabetes to keep their fasting blood glucose between $3.5 \mathrm{mmol} / \mathrm{l}$ and $5.9 \mathrm{mmol} / \mathrm{l}$ and their one hour postprandial blood glucose below $7.8 \mathrm{mmol} / \mathrm{l}$ if this is safely achievable.

The safety of medications for diabetes and its complications before and during pregnancy

- Metformin may be used for women with preexisting diabetes as an adjunct or alternative to insulin in the preconception period and during pregnancy; discontinue all other oral hypoglycaemic agents before pregnancy and substitute with insulin.

- The rapid acting insulin analogues aspart and lispro are safe to use during pregnancy; isophane insulin is the first choice for long acting insulin during pregnancy.

- Discontinue angiotensin converting enzyme inhibitors and angiotensin II receptor antagonists before pregnancy or as soon as pregnancy is confirmed; substitute with alternative antihypertensive agents suitable for use during pregnancy. 
- Discontinue statins before pregnancy or as soon as pregnancy is confirmed.

\section{Management of diabetic emergencies and complications during pregnancy}

- Advise women with insulin treated diabetes of the risks of hypoglycaemia and of hypoglycaemia unawareness during pregnancy (particularly in the first trimester); provide a concentrated glucose solution (and glucagon for women with type 1 diabetes) and advise women and their partners or other family members on their use.

- Exclude diabetic ketoacidosis as a matter of urgency in women with type 1 diabetes who become unwell during pregnancy; admit women who are suspected of having diabetic ketoacidosis immediately for level 2 critical care, where they can receive medical and obstetric care.

- Offer retinal and renal assessment to women with pre-existing diabetes before and during pregnancy because diabetic retinopathy may worsen during pregnancy and diabetic nephropathy is associated with adverse pregnancy outcomes (such as intrauterine growth restriction, preeclampsia, and preterm birth).

\section{Gestational diabetes}

- Use risk factors (raised body mass index, previous macrosomic baby, previous gestational diabetes, family history of diabetes, and family origin with a high prevalence of diabetes) to decide which pregnant women to test for gestational diabetes. In these women test for gestational diabetes using a two hour $75 \mathrm{~g}$ oral glucose tolerance test (OGTT) and the World Health Organization's diagnostic criteria for gestational

\section{Effects of diabetes on pregnancy and effects of pregnancy on diabetes}

\section{Information and advice for women with diabetes and their families should cover the} following points

- The role of diet, weight, and exercise (including weight loss advice for women who have a body mass index $\left(\mathrm{kg} / \mathrm{m}^{2}\right)$ above 27$)$

- The increased risk of having a baby who is large for gestational age, increasing the likelihood of birth trauma, induction of labour, and caesarean section

- The importance of maternal glycaemic control during labour and birth and early feeding of the baby to reduce the risk of neonatal hypoglycaemia

- The possibility of transient morbidity (such as hypoglycaemia or respiratory distress syndrome) in the baby during the neonatal period, which may require admission to a neonatal unit

- The risk of the baby developing obesity and/or diabetes in later life

For women with pre-existing diabetes information and advice should also cover the following points

- The need for folic acid supplementation (5 mg a day) until 12 weeks' gestation to reduce the risk of having a baby with a neural tube defect

- The risks of hypoglycaemia and of hypoglycaemia unawareness during pregnancy and the effects of nausea and vomiting during pregnancy on glycaemic control

- The need for assessment of diabetic retinopathy and nephropathy before and/or during pregnancy. diabetes. In women who have had gestational diabetes in a previous pregnancy, offer early self monitoring or an OGTT at 16-18 weeks and a further OGTT at 28 weeks if the results are normal; in women with any of the other risk factors for gestational diabetes offer an OGTT at 24-28 weeks. Do not screen using fasting plasma glucose, random blood glucose, glucose challenge test, or urine analysis for glucose. ${ }^{4}$

- Inform women with gestational diabetes about the effects of gestational diabetes on pregnancy (box); tell them that good glycaemic control throughout pregnancy will reduce the risk of fetal macrosomia, trauma during birth (to themselves and the baby), induction of labour or caesarean section, neonatal hypoglycaemia, and perinatal death; and instruct them in self monitoring of blood glucose. Targets for blood glucose control are the same as for women with pre-existing diabetes.

- Consider hypoglycaemic therapy if diet and exercise fail to maintain blood glucose targets during a period of one to two weeks; also consider hypoglycaemic therapy if ultrasound investigation suggests incipient fetal macrosomia (abdominal circumference above the 70th centile).

- Tailor hypoglycaemic therapy for women with gestational diabetes to the glycaemic profile of and acceptability to the individual woman (hypoglycaemic therapy comprises one or more of: regular insulin, the rapid acting insulin analogues lispro and aspart, the hypoglycaemic agents metformin and glibenclamide).

\section{Antenatal care appointments}

- Offer pregnant women with diabetes immediate contact with a joint diabetes and antenatal clinic; contact with the diabetes care team for assessment of glycaemic control should occur every one to two weeks; antenatal appointments should cover care specifically for women with diabetes in addition to the care provided routinely for healthy pregnant women.

- Prioritise women with diabetes for examination of the four chamber view of the fetal heart and outflow tracts at $18-20$ weeks.

\section{Preterm labour in women with diabetes}

- Antenatal steroids for fetal lung maturation are not contraindicated but should be administered with additional insulin in women with insulin treated diabetes.

- Tocolysis is not contraindicated, but do not use betamimetics for tocolysis in women with diabetes.

\section{Timing and mode of birth}

- Offer an anaesthetic assessment in the third trimester to women with comorbidities such as obesity or autonomic neuropathy; if general anaesthesia is used for the birth, monitor blood glucose every 30 minutes from induction of 
general anaesthesia until after the baby is born and the woman is fully conscious.

- Offer elective birth after 38 completed weeks (induction of labour or, if indicated, elective caesarean section); inform women who have a macrosomic fetus that has been diagnosed with ultrasound about the risks and benefits of vaginal birth, induction of labour, and caesarean section.

\section{Glycaemic control during labour and birth}

- Monitor capillary blood glucose hourly during labour and birth to maintain a concentration between $4 \mathrm{mmol} / \mathrm{l}$ and $7 \mathrm{mmol} / \mathrm{l}$; if the concentration is not maintained in this range, use intravenous dextrose and insulin infusion during labour and birth.

- In women with type 1 diabetes, consider intravenous dextrose and insulin infusion from the onset of labour.

Initial assessment and care of the newborn baby

- Advise women to give birth in hospitals where advanced neonatal resuscitation skills are available 24 hours a day; keep babies with their mothers unless a clinical complication or abnormal clinical signs arise that warrant admission for intensive or special care.

- Feed babies as soon as possible after birth (within 30 minutes) and then every two to three hours until feeding maintains prefeed blood glucose concentrations of at least $2.0 \mathrm{mmol} / \mathrm{l}$. Test blood glucose concentrations at two to four hours after birth. Only if concentrations are below $2.0 \mathrm{mmol} / \mathrm{l}$ on two consecutive readings despite maximal support for feeding, or if there are abnormal clinical signs, or if the baby will not feed orally effectively, should additional measures such as tube feeding or intravenous dextrose should be given. Test blood glucose in babies who present with clinical signs of hypoglycaemia (such as abnormal muscle tone or level of consciousness, fits, apnoea) and start treatment with intravenous dextrose as soon as possible.

- Perform echocardiography for babies who show clinical signs associated with congenital heart disease (including heart murmur) or cardiomyopathy.

- Do not test for polycythaemia, hyperbilirubinaemia, hypocalcaemia, or hypomagnesaemia unless the baby has clinical signs.

- Recognise criteria for admission to a neonatal unit, such as hypoglycaemia associated with abnormal clinical signs, respiratory distress, signs of cardiac decompensation, or neonatal encephalopathy.

\section{Postnatal management of diabetes}

Pre-existing diabetes

- Reduce insulin immediately after the birth in women with insulin treated pre-existing diabetes.
Monitor blood glucose levels carefully to establish the appropriate dose, and inform women of the increased risk of hypoglycaemia in the postnatal period, especially if breast feeding (when a meal or snack before or during feeds is advisable).

- Resume (or continue) use of metformin and glibenclamide immediately after birth in women with pre-existing type 2 diabetes who are breast feeding.

- Continue to avoid any drugs for treating diabetes complications that were discontinued for safety reasons in the pre-conception period.

- Refer women with pre-existing diabetes back to their routine diabetes care arrangements; remind them of the importance of contraception and the need for preconception care when planning future pregnancies.

\section{Gestational diabetes}

- Discontinue hypoglycaemic therapy immediately after the birth in women who were diagnosed with gestational diabetes; test their blood glucose to exclude persisting hyperglycaemia before transfer to community care, and remind them of the symptoms of hyperglycaemia.

- Offer lifestyle advice (on weight control, diet, and exercise) and a fasting plasma glucose measurement (but not an OGTT) at the six week postnatal check and annually thereafter.

- Provide information about the risk of gestational diabetes in future pregnancies, offer screening for diabetes when planning future pregnancies, and offer early self monitoring of blood glucose or an OGTT in future pregnancies.

\section{Overcoming barriers}

This guidance for care of women with diabetes and their families aims to empower them so that pregnancy and child birth are positive experiences. For example, uptake of preconception care is encouraged by using every contact with healthcare professionals (including the diabetes care team) to inform women of childbearing age about the benefits of preconception glycaemic control and every contact with the diabetes care team to document intentions about pregnancy and contraceptive use, and by also giving preconception care in a supportive environment, with the woman's partner or other family members encouraged to attend. Other recommendations aim to prepare women for the additional time and effort required to manage diabetes during pregnancy and to provide maximal support for women who wish to breast feed.

To support implementation, NICE and the guideline development group have developed a version of the guidance (available at www.nice.org.uk/CG063Publi cInfoEnglish) that can be given to women with preexisting diabetes, women who are planning to become pregnant, and women's partners or families. 
Contributors: Moira A Mugglestone, Anuradha Sekhri, and Robert Fraser wrote the initial draft of the article using material produced collectively by the entire guideline development group. They also contributed to its revision and the final draft, having received feedback from every member of the group.

Funding: The National Collaborating Centre for Women's and Children's Health was commissioned and funded by the National Institute for Health and Clinical Excellence to write this summary.

Competing interests: Several members of the guideline development

group have declared competing interests-please see the version of this article on bmj.com.

Provenance and peer review: Commissioned; not externally peer reviewed.
1 Casson IF. Outcomes of pregnancy in insulin dependent diabetic women: results of a five year population cohort study. BMJ 1997;315:275-8.

2 Hawthorne G. Prospective population based survey of outcome of pregnancy in diabetic women: results of the northern diabetic pregnancy audit, 1994. BM/ 1997;315:279-81.

3 National Institute for Health and Clinical Excellence. Diabetes in pregnancy: management of diabetes and its complications from pre conception to the postnatal period. London: NICE, 2008. www.nice.org.uk/CG063

4 World Health Organization Department of Noncommunicable Disease Surveillance. Definition, diagnosis and classification of diabetes mellitus and its complications. Report of a WHO consultation. Part 1: diagnosis and classification of diabetes mellitus. Geneva: WHO, 1999.

\title{
Commentary: Controversies in management of diabetes from preconception to the postnatal period
}

\author{
Lucy C Chappell,' Sarah J Germain²
}

${ }^{1}$ Division of Reproduction and Endocrinology, King's College London, London SE1 7EH

${ }^{2}$ Department of Diabetes and Endocrinology, Guy's and St Thomas' NHS Foundation Trust, London SE1 7EH

Correspondence to: Lucy C Chappell lucy.chappell@kcl.ac.uk

BMJ 2008;336:717-8 doi:10.1136/bmj.39518.615058.AE
Why does the United Kingdom remain so far from achieving the aim of "a pregnancy outcome for women with diabetes that equates with that of women without diabetes," an aim outlined at the landmark St Vincent meeting of diabetes experts, government representatives, and patient organisations nearly 20 years ago? ${ }^{1}$ Pregnancy outcomes in the UK continue to be substantially worse for women with pre-existing diabetes (both type 1 and type 2), with rates of congenital anomalies and perinatal mortality significantly higher than in non-diabetic women and with care regularly falling short of the required standard. ${ }^{2}$ Many of the recommendations in the new guidelines from the National Institute for Health and Clinical Excellence (NICE) seem sensible and will be of use to all healthcare professionals in this area, but in places they are surprisingly clear cut given the relative paucity of high quality evidence.

The guidelines suggest a comprehensive programme of care from preconception to postnatal management. They include a strong emphasis on preparation for pregnancy, which requires considerable involvement of general practitioners, diabetologists, specialist obstetricians and midwives, and most importantly the women themselves. This message has been echoed by the latest triennial maternal mortality report from the UK, which highlights the importance of joined-up care for such women. ${ }^{3}$ Given that two thirds of pregnant women with pre-existing diabetes had a haemoglobin $\mathrm{A}_{1 \mathrm{C}}$ concentration greater than $7 \%$ in the first trimester (the threshold for optimal glycaemic control in the report from the Confidential Enquiry into Maternal and Child Health), ${ }^{2}$ achieving the recommendation of aiming to "maintain a $\mathrm{HbA}_{1 \mathrm{C}}$ below $6.1 \%$ " seems particularly optimistic. Much greater uptake of structured education programmes and use of continuous subcutaneous insulin infusion pumps, as shown to be beneficial outside pregnancy, may need to be considered to achieve these tough targets. The resource implications should not be underestimated, particularly when those with poorest control may find it hardest to access and use health care.

The recommendation that screening for gestational diabetes should be by clinical risk factor alone is more controversial. The authors acknowledge the poor sensitivity and specificity of this approach compared with universal administration of oral glucose tolerance tests. In some groups, use of clinical risk factors misses nearly half the women with gestational diabetes, ${ }^{4}$ but the performance of this screening method will depend on the maternal age, ethnicity, and body mass index profile of each population. As the ACHOIS trial ${ }^{5}$ provided evidence of potential benefit for treatment of even mild gestational diabetes, missing a substantial proportion of cases of gestational diabetes may translate into clinical detriment. The ongoing hyperglycaemia and pregnancy outcome trial (which is assessing associations of maternal glycaemia with risks of adverse pregnancy outcome) will clarify further the need for treatment in this milder group. On a positive note, the option to use metformin for the treatment of gestational and type 2 diabetes will be welcomed as an alternative or addition to insulin.

Obstetricians may be surprised, given the limited evidence, at the recommendation to offer delivery to all women with diabetes at 38 weeks' gestation as this will represent a change in clinical practice for many. It reflects the difficulty with which late pregnancy complications, particularly shoulder dystocia and stillbirth, can be predicted. Women with diabetes already have a caesarean section rate of $60-70 \%$, three times higher than the rate in the general population of women giving birth; the debate continues about the most appropriate rate for caesarean section in diabetic women, given the short and long term risks of this procedure, particularly in obese women. Optimising glycaemic control to prevent macrosomia and understanding the mechanism by which diabetes increases the risk of stillbirth may be more thoughtful approaches in the long term. 